\title{
Expression of Concern to: Composite recurrent Laguerre orthogonal polynomials neural network dynamic control for continuously variable transmission system using altered particle swarm optimization
}

\author{
Chih-Hong Lin
}

Published online: 22 February 2021

(C) Springer Nature B.V. 2021

\author{
Abstract The Editor-in-Chief is issuing an Editorial \\ Expression of Concern for this Article. \\ Expression of Concern to: \\ Nonlinear Dyn (2015) 81:1219-1245 \\ https://doi.org/10.1007/s11071-015-2064-7
}

The Editor-in-Chief is issuing an Editorial Expression of Concern for this Article. It has been brought to the journal's attention that there is significant overlap with a number of articles which were under consideration at the same time, but were published after this article [1-9] (amongst others).

Author C.H. Lin has not provided a clear response regarding this Editorial Expression of Concern.

\section{References}

1. Lin, C.H.: Novel application of continuously variable transmission system using composite recurrent Laguerre orthogonal polynomials modified PSO NN control system. ISA Trans. 64, 405-417 (2016)

2. Lin, C.H.: Application of v-belt continuously variable transmission system using hybrid recurrent Laguerre orthogonal

The original article can be found online at https://doi.org/10. 1007/s11071-015-2064-7..

C.-H. Lin $(\varangle)$

Department of Electrical Engineering, National United

University, No. 2, Lienda, Nan-Shi Li, Miaoli 36003,

Taiwan

e-mail: jhlin@nuu.edu.twgh

URL: http://www.ee.nuu.edu.tw/03_professor/024/tirepage/t06.htm polynomials neural network control system and modified particle swarm optimization. J. Comput. Nonlinear Dyn. 10(5), (2015)

3. Lin, C.H.: Hybrid recurrent Laguerre-orthogonal-polynomial NN control system applied in V-belt continuously variable transmission system using particle swarm optimization. Math. Probl. Eng. (2015)

4. Lin, C.H.: Application of hybrid recurrent Laguerreorthogonal-polynomial $\mathrm{NN}$ control in V-belt continuously variable transmission system using modified particle swarm optimization. J. Mech. Sci. Technol. 29, 3933-3952 (2015). https://doi.org/10.1007/s12206-015-0839-x

5. Lin, C.H.: Hybrid recurrent Laguerre-orthogonalpolynomials neural network control with modified particle swarm optimization application for V-belt continuously variable transmission system. Neural Comput. Appl. 28, 245-264 (2017). https://doi.org/10.1007/s00521-015-2053-2

6. Lin, C.H.: Application of a V-belt continuously variable transmission system by using a composite recurrent Laguerre orthogonal polynomial neural network control system and modified particle swarm optimization. J. Vib. Control 23(9), 1437-1462 (2017)

7. Lin, C.H.: Design of a composite recurrent Laguerre orthogonal polynomial neural network control system with ameliorated particle swarm optimization for a continuously variable transmission system. Control Eng. Pract. 49, 42-59 (2016)

8. Lin, C.H.: An intelligent dynamic control of continuously variable transmission system using modified particle swarm optimization. Proc. Inst. Mech. Eng. Part C J. Mech. Eng. Sci. 230(13), 2181-2207 (2016)

9. Lin, C.H.: A six-phase CRIM driving CVT using blend modified recurrent Gegenbauer OPNN control. J. Power Electron. 16(4), 1438-1454 (2016)

Publisher's Note Springer Nature remains neutral with regard to jurisdictional claims in published maps and institutional affiliations. 